\title{
Magnitude and associated factors of cutaneous leishmaniasis; in Mekelle city, Ayder referral hospital, Tigray, Northern Ethiopia, 2014
}

\author{
Feleke Tilahun ${ }^{1}$, Workalemahu Alemu ${ }^{2}$, Getasew Mulatu ${ }^{3}$ \\ ${ }^{1}$ Feleke Tilahun Zewdu, Mekelle University, Post Graduate in Tropical Dermatology, Mekelle, North Ethiopia \\ ${ }^{2}$ Workalemahu Alemu, Mekelle University, Dermato-Venerology Department, Mekelle, North Ethiopia \\ ${ }^{3}$ Getasew Mulatu, Mekelle University, Ayder Referral hospital, head nurse, Mekelle, North Ethiopia
}

Email address:

momflk@gmail.com (F. Tilahun),work2012@gmail.com (W. Alemu), getasewmulatu@gmail.com (G. Mulatu)

\section{To cite this article:}

Feleke Tilahun, Workalemahu Alemu, Getasew Mulatu. Magnitude and Associated Factors of Cutaneous Leishmaniasis; in Mekelle City, Ayder Referral Hospital, Tigray, Northern Ethiopia, 2014. Clinical Medicine Research. Vol. 3, No. 6, 2014, pp. 189-199.

doi: $10.11648 /$ j.cmr.20140306.16

\begin{abstract}
Introduction; Cutaneous leishmaniasis is a neglected tropical disease strongly associated with poverty with an adverse health effects over the poorest populations of the world. The disease is endemic in 88 countries, 72 of them are developing countries including Ethiopia. It has been estimated that 12 million people are infected and 350 million people are at risk with the annual incidence of new cases is estimated between 1.5-2million. Ethiopia has seen new outbreaks in areas previously not known to be endemic often with co-infection by the human immunodeficiency virus with rates reaching $5.6 \%$ of the cases and other associated risk factors. As best of my knowledge similar study was not conducted specifically in Mekelle even though other study conducted to the whole region, Tigray. Thus, this preliminary study concerns to determine the magnitude and associated factors of cutaneous leishmaniasis in Mekelle city.Objectives; To assess the prevalence and associated factors of cutaneous leishmaniasis on patients who visit dermatology out patient department in Ayder referral hospital, Mekelle, Tigray, Northern Ethiopia. Methods and subjects; Institutional based cross sectional study was conducted on 478 study population from Ayder referral hospital by using systematic random sampling technique. Data entry and analysis was conducted by using SPSS v-20. The association between different variables in relation to the outcome variable was measured by odds ratio with $95 \%$ confidence interval. All variables with $\mathrm{P}<0.05$ in the bivariate analysis were included in the multivariate model, accepting $\mathrm{P} \leq 0.05$ as the inclusion level for the independent variables. Result; Prevalence of cutaneouse leishmaniasis in this study was nearly six percent (5.6\%) with 99\% response rate. Sex [AOR 2.1(95\% CI); (0.15, 0.79)], income source being farmer [AOR 2.1(95\% CI); (0.00.0.248)], location of the home near to both farm and gorge [AOR 10.1 $(95 \% \mathrm{CI}) ;(0.00,0.574)]$ and presence of the hyrax [AOR $1.1(95 \% \mathrm{CI}) ;(0.00,0.60)$ ] were statistically significant to the presence of cutaneouse leishmaniasis in Mekelle city. Conclusion The overall prevalence of cutaneouse leishmaniasis in the study area was relatively high $(5.6 \%)$ having a statistical significant association with sex, income source (farmer), location of the residence near to farm land and gorge and the presence of hyrax. Thus, a continuous health education and awareness creation about the disease, factors and preventive methods of cutaneouse should be sought.
\end{abstract}

Keywords: Magnitude, Cutaneouse Leishmaniasis, Mekelle City

\section{Introduction}

\subsection{Background}

Leishmaniasis is a group of diseases caused by obligatory and intracellular haemoflagellate protozoan parasites of the genus Leishmania. Human leishmaniasis is a complex disease with many clinical forms, which range from mild self-healing cutaneous lesions to fatal visceral disease. Leishmaniasis, a major global health and economic issue, comprises a group of diseases that transmitted to mammals, including humans, requires the bite of female phlebotomine sand flies (1-3). 
Cutaneous leishmaniasis (CL) is a neglected tropical disease strongly associated with poverty with an adverse health effects over the poorest populations of the world which is highly increases its burden as a result of increasing migration, regional climatic change, and impaired immunity resulting from malnutrition and/ or HIV/AIDS (4).

The health situation of Africa continent is characterized by immense disease burden and weak health systems embedded by the context of poverty, under development and conflicts. Ethiopia is not unique in this perspective as the case is testified by its poor health outcomes even by sub-Saharan Africa's standards. Consequently, there is the dissemination of various infectious diseases across the country due to the low spending of the government health care financing and the minimal participation of the private sector. With this regard the category of neglected tropical diseases that affect almost exclusively the poorest populations have been given lesser attention and overshadowed by the major focus of HIV/AIDS, Tuberculosis and Malaria. A case in point, cutaneous leishmaniasis is one of the infections, which share neither the public eye nor better financial returns amongst the neglected tropical diseases $(5,6$, and 7$)$.

The disease is endemic in 88 countries, 72 of them are developing countries. It has been estimated that 12 million people are infected and 350 million people are at risk with the annual incidence of new cases is estimated between 1.52million $(8,9,10)$.

The global epidemiology of cutaneous leishmaniasis has changed; which is characterized by emergence and reemergence in many parts of the world, ascribed to population movement, man-made environmental changes, AIDS and other immunosuppressive conditions. Furthermore, an epidemiological pattern of leishmaniasis is in evolution because of an accumulation of risk factors, like immunesuppression, human and environmental changes and drug resistance. The epidemiology of leishmaniasis in a given area is directly dependent on the behavior of the human and/or animal population in relation to the cycle of transmission. There are a variety of factors that influence the risk of transmission of cutaneous leishmaniasis. Some of these factors include proximity of residence to sand fly breeding, resting sites, type of housing, occupation, extent of exposure to sand fly bites, natural resistance (genetic or acquired) to infection, virulence of the parasite species and others $(4,11)$.

Leishmaniasis has been an ancient public health problem in South-West Asia and the Arab World reported from time immemorial as the pharaohs ruled in Egypt and Assyrians in Mesopotamia. It was extensively described by Arab-Islamic scientists like Avicenna (IbnSina, 980-1037 A.D.) who wrote a complete chapter in his prominent book entitled AlkanounFi E1 Tebb raising the possibility of mosquitoes being involved in the transmission of the disease. He has described cutaneous leishmaniasis (CL) as a disease endemic in Balk (Afghanistan) and Baghdad (2, 4, 12).

Leishmania HIV co-infection has emerged because of the increasing overlap between HIV and Leishmania. Cases of co-infection have been reported from 36 countries and the co-infection is increasing especially in Southern Europe and Africa. Co-infection with HIV has led to the spread of leishmaniasis, typically a rural disease, into urban areas. By far, it is considered as the fifth opportunistic disease (13-16).

CL was first described in Ethiopia by an Italian epidemiologist Martogilo in 1913. CL is known by different vernacular name in different localities of Ethiopia such as: "Volbo" in Ocholo, "Finchoftu" in central Shoa, "Kunchir" in Gojam, Gonder and parts of Wollo, "Giziwa" in Tigray, "Chewie" in Sodo, "Simbirahalkani" in Wollega and "Shahegne" in north Shewa $(4,17)$.

Ethiopia is one of the endemic areas for leishmaniasis in the world. The health status in Ethiopia is poor and the population of Ethiopia faces high morbidity and mortality rates $(4,8,10)$.

The disease is found mainly in high and mid-altitudes ranging between 1,400 to 2,700 meters above sea level. However, L. tropical isolated from sand flies in low altitude of the Awash Valley (about 900 meters above sea level). From the same area, isolated and described L. tropica from one patient who had lived in the area (11).

Leishmaniasis is a climate-sensitive disease, occupying a characteristic climate space that is strongly affected by changes in rainfall, atmospheric temperature and humidity.

Seasonal labor movements may also spread the disease, with the return of migrants to non-endemic areas, as appears to have occurred in the highlands of Ethiopia in the 2000s. Behavior, such as sleeping outside under acacia trees and living in houses constructed of grassy material, appears to increase risk for the disease $(18,19,20)$.

\subsection{Statement of the Problem}

Cutaneous leishmaniasis is one of the widespread tropical infectious diseases. It affects thousands of people in Ethiopia. Though, the magnitude of the disease in the country is not well documented, recently the presence of CL in Tigray region including Mekelle city has been reported $(19,21)$. This indicates the need for epidemiological surveillance of the disease in Tigray region, in particular, and in the country in general.

Moreover, a single study was conducted in Tigray region, but to the best of my knowledge there research conducted specifically in Mekelle city. So that I developed the interest to conduct such an open up study to address the gap. Finally, I have explored the major determinant factors with describing the magnitude of the disease in Mekelle city with identifying the possible co-morbidities, socio-economical and socio-demographic aspects of cutaneous leishmaniasis in line of time, person, area and others patterns.

\subsection{Significance of the Study}

This study provides a basic information on the prevalence and associated factors of $\mathrm{CL}$ that have a valuable contribution for the program planners, the academic community, service providers, health care professionals and above all patients suffering from CL will be beneficiary from 
this study as prevention and early detection which would to lower morbidity due to this debilitating, preventable and curable disease.

\section{Literature Review}

Neglected tropical diseases have made adverse health effects over the poorest populations of the world. It is estimated that 1billion people suffer from these diseases worldwide and most cases are found in Africa (20, 21, and 22).

\section{Magnitude of the Problem}

There are 1.0-1.5 million cases of CL each year, with $90 \%$ of CL cases occurring in 8 countries: Afghanistan, Algeria, Brazil, Iran, Peru, Saudi Arabia and Syria, showing that the Middle East is a central focus for CL $(15,21)$.

The ten countries with the highest estimated case counts, Afghanistan, Algeria, Colombia, Brazil, Iran, Syria, Ethiopia, North Sudan, Costa Rica and Peru, together account for 70 to $75 \%$ of global estimated CL incidence $(4,21,22)$.

Many research reviews showed that the prevalence of cutaneous leishmaniasis was in Ochollo the overall prevalence of cut leishmaniasis was 3.6-4.0\%, in Sebeta, 25 $\mathrm{km}$ south west of Addis Ababa and found that the prevalence of CL with active infection was $0.9 \%$ (9/1,000), and prevalence of active CL in Siliti woreda was $4.82 \%(4,9)$.

Socio-Demographic Factors

A study in India showed that $75.93 \%$ cases were of males, $24.07 \%$ cases were of females and the male to female ratio was $3: 1$. The majority of cases were found to be of patients aged between 15 to 45 years of age i.e., 64.67 and $21.80 \%$ cases respectively, children below 14 years of age had the least number of cases where as patients more than 45 years of age i.e., $13.53 \%$. Almost, majority of cases belong to the poor people of rural areas of Bihar, India (24).

Other similar study in Jordan showed that highest infection rate of $72.4 \%$ was recorded in males compared to $27.6 \%$ in females $(25)$.

A study conducted in America showed no significant difference was found between males and females suggesting that they are equally exposed to infection (13).

A study conducted in Silti zone, Ethiopia, reveals that the mean age for the CL cases was 17.9 years $\pm 1.5 \mathrm{SE}$ (standard error) with a range of 2-70 years. There was no gender difference in the frequency of CL cases, $47.8 \%$ (44/92) male vs. $52.2 \%$ (48/92) female. Besides, from host factors tested in the analysis, age and the educational status of the head of the household were associated with the risk of development of CL (4).

Similar study which were conducted in Tigray region, reveals that the mean age was 23.7 years (99\% CI: 21.8-25.6) and $60 \%(n=282)$ of patients were in the age group 15-44 years; $28 \%$ of patients were under 14 years of age with a prevalence of CL in Tigray was $4.8 \%$. Meanwhile, the male to female ratio of patients who have affected was 2.5:1; $71.1 \%$ of patients were males. A male prevalence was observed both in children and adults with $73.7 \%$ and $68.4 \%$ respectively (17).

Most reviews showed that the affected populations are mainly very poor male seasonal migrant workers that travel in the harvesting season from non-endemic highlands to the cotton, sesame and sorghum fields of Humera and Metema on the Sudanese border $(17,28)$. Similarly, a review conducted in Bolivia, $25 \%$ of the population showed active or healed lesions of cutaneous leishmaniasis within 3 years of travel or movement to somewhere else which is endemic for cutaneous leishmaniasis (20).

A review in Colombia showed from those patients of cutaneous leishmaniasis the use of bed nets estimated $63 \%$ (258/409) of people who lived in houses with bed nets slept under a net (23).Similarly WHO reports as well as recommends that cutaneous leishmaniasis can be prevented or could be minimized using appropriate preventive methods such as bed nets, using spray insecticides, repellents and long sleeves (22).

A review from London and American family of physicians (2004) recommend as the use of insecticides in endemic areas is important for travelers. House and space spraying have reduced sand fly populations, and fine-weave pyrethroid-impregnated bed-nets have been used in Burkina Faso, Sudan, and Columbia. Destruction of rodent reservoirs by pumping insecticides into rodent burrows has had limited success (14).

A research conducted in Ecuador memorial institute showed that the socio-economic status was determined to be cement floors $(32 \%, 148 / 463)$, tiled floors $(19 \%, 89 / 463)$, and ownership of cows $(58 \%, 266 / 463)$ horses (24\%, $112 / 463)$, or pigs $(75 \%, 346 / 463)$. Soil floors $(29 \%, 134 / 463)$ (12).

\section{Clinical Spectrum CL}

A study conducted in India showed that up to $7 \%$ of treated and cured kala-azar cases developed PKDL. In some cases PKDL developed without the patient suffering from clinical kala-azar (24). Similar study in Sudan shows that the lesions were found on different parts of the body including the limbs $(87.5 \%)$, face $(1.4 \%)$, and trunk (1.4\%).Totally, $87.5 \%$ of the cases had at least one lesion on the lower or upper limbs (26).

On the other hand, in Palestine reveals that individuals who had skin lesions, $89.1 \%(82 / 92)$ had the lesions on their face, mostly on the check or the nose. The face is the exposed body part that is easily accessible to sand fly bites. In addition, the softness of the skin on the face makes it a preferable site of feeding for the sand fly (27).

A study done in Silti zone, Ethiopia, reveals that most of the patients, $46.7 \%$ (43/92), had single lesions and only $17.4 \%$ (16/92) developed three or more. Three quarters of the patients (69/92) had a lesion that had developed six or more months before starting treatment. More than half of the CL cases, 55.4\% (51/92), developed lesions on either or both cheeks. Lesions were also common on the nose $13.0 \%$ (12/92). Among all the CL confirmed cases $19.2 \%$ (14/73) were clinically of the muco-cutaneous type. The presence of a scar was registered in only $0.3 \%$ (6/1907) of the 
participants (4) where as in Ochello it was found to be $34.4 \%$ (5).

Similar study done in Tigray, Ethiopia states a wide spectrum of clinical presentations was seen; $86 \%(n=405$; 99\% CI: 82.5-89.0) of patients had localized cutaneous leishmaniasis (LCL), mucosal lesions were observed in $11 \%$ of cases $(\mathrm{n}=52 ; 99 \% \mathrm{CI}$ : 8.4-14.2), another $2.3 \%(\mathrm{n}=11$; 99\% CI: $\left.1.2^{\wedge} .1\right)$ of cases had the clinical pattern of DCL and only $0.7 \%(\mathrm{n}=3 ; 99 \% \mathrm{CI}: 0.1-1.9)$ presented with PKDL (17).

\section{HIV/AIDS co-infection}

Co-infection of HIV and Leishmaniasis produces cumulative deficiency of host CMI, a key factor for primary protection against infection, recurrences or spread of parasites. An HIV-infected patient with CL may display an atypical and severe clinical presentation of CL in terms of number (>200), sites and types of lesions. Uncommon sites of infection are more frequent, such as the gastrointestinal tract or the upper respiratory tract (22).

Among CL-infected individuals in Cameron, $4.8 \%$ were co-infected with HIV; both HIV serotypes (HIV-1 and HIV-2) were recorded (22).

A review in Ethiopia showed that out of 186 patients who underwent VCT, 15 patients (8.1\%; 99\% CI: 4.6-13.0) resulted as co-infected and their clinical appearance was characterized by destroying muco-cutaneous lesions, multiple locations, atypical manifestations and a relapsing course. Besides, leishmania HIV co-infection has emerged because of the increasing overlap between HIV and Leishmania. Cases of co-infection have been reported from 36 countries and the co-infection is increasing especially in Southern Europe and Africa $(11,17)$.

On otherways, a review in Ethiopia, Sililti zone, showed that the proportion of CL cases in the age group of 11-20 years old was $8.3 \%$ and $44.6 \%$ in Ocholo and Silti respectively (4).

Geographical Factors

The geographic spread of CL is due to factors related mostly to development. These include massive rural-urban migration and agro-industrial projects that bring non-immune urban dwellers into endemic rural areas. Man-made projects with environmental impact, like dams, irrigation systems and wells, as well as deforestation, also contribute to the spread of leishmaniasis $(4,28)$.

A review in Brazil showed that the development of new settlements, intrusion into primary forest, deforestation, massive migration from rural to urban areas, rapid and unplanned urbanization, the building of dams and new irrigation schemes are primary risk factors for the spread of leishmaniasis $(14,29)$.

Similarly in Ethiopia review points out rainfall have a slight, non significant effect on the disease risk: by matching 'low rain' highlands with 'high rain' highlands, the possibility of being affected by leishmaniasis was only slightly increased. People living in highlands with a high precipitation rate have a 3.6 prevalence risk ratio of leishmaniasis (99\% CI: 2.2-5.8) versus those living in the lowlands, as well as the same ratio of the population living in highlands (5).

Similar study in Silti zone, Ethiopia, reveals that the environmental factors: the distance of the gorge from the participant's house, Acacia spp and hyrax near the participant's house, the use of impregnated bed nets and possessing domestic animals were found to be associated with the development of CL. Forests in general have shrunk from covering $65 \%$ of the country and $90 \%$ of the highlands to $2.2 \%$ and $5.6 \%$ respectively. Such changes will undoubtedly influence the profile of vector borne diseases $(17,21)$.

The presence of the gorge around $(<300 \mathrm{~m})$ far away from the participant's house had a significant association to acquire CL with the AOD of 1.762 (2.600-5.179)(4). Other study conducted in America, Argentina (Northwest America) reveals the presence of a pond or gorge or woodland within $150 \mathrm{~m}$ of the house and an agricultural area within $200 \mathrm{~m}$ of the house had a significant association to acquire CL, gorge with AOD of $15.1(6.1-7.3)$ and cultivating farm with 2.6 $(1.2-5.5)(12,15,22)$

Studies conducted by Edessa, et al. suggest that the disease is found mainly at high and mid altitudes ranging from 1400 to $2700 \mathrm{~m}$ above sea level, which is most favorable for the proven vector sand flies species of phlebotomus longipes and P. Pedifer (vectors). A lower level altitudinal limit of $1200 \mathrm{~m}$ also suspected to be the other potential area of Cutaneous Leishmaniasis distribution. Similarly most of patients $(n=166)$ were from Mekelle and the nearby villages in the Tembien mountains. The case prevalence was higher in the mountain districts than in the Valleys. Males living at altitudes above 2000m ASL represented the Leishmania cases matched by altitude and gender (4).

\subsection{Conceptual Frame Work}

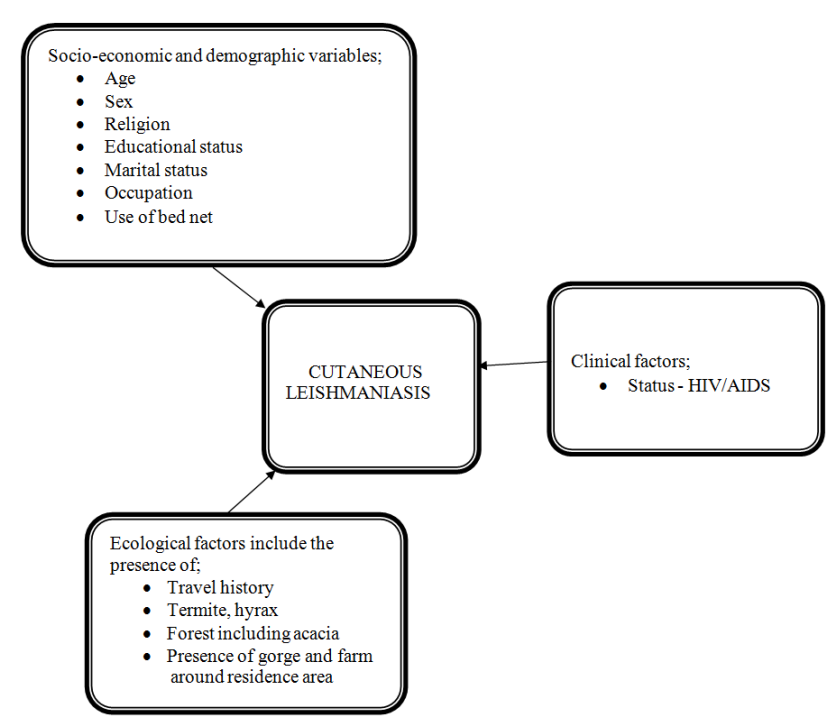

Figure 1. Shows Conceptual framework (Adopted from Dawit G, 2013 \& E. Negera et al.2008) $(4,30)$ 


\section{Objectives}

\subsection{General Objective}

To assess the prevalence and associated factors of cutaneous leishmaniasis on patients who visit dermatology department in Mekelle city, Ayder referral hospital, Tigray, Ethiopia.

\subsection{Specific Objectives}

- To assess the prevalence of cutaneous leishmaniasis on patients who visit dermatology department in Ayder referral hospital.

- To determine the possible factors for cutaneous leishmaniasis on patients who visit dermatology department in Ayder referral hospital.

\section{Method and Materials}

\subsection{Study Area and Period}

Mekelle, the capital city of the Tigray National Regional State, lies $780 \mathrm{~km}$ north of Addis Ababa. The city is located at $39^{0} 33$ 'E longitude and $13^{0} 32$ ' $\mathrm{N}$ latitude, situated in the extension of the central highlands of Ethiopia. The altitude of Mekelle is between $1965 \mathrm{~m}$ and $2220 \mathrm{~m}$ above sea level. The city is bounded by mountain ranges in the east and north with a notable physical feature of hills and valleys $(19,31)$.

Mekelle city has 4 governmental (1 referral) and 2 private hospitals, 8 governmental health centers, 32 private clinics, 3 dental private clinics, 5 private diagnostic laboratories, 32 private pharmacies, 2 private dermato-venereology clinics and 3 private physiotherapy centers which serves for a total of Mekelle residents 227,505 and the surrounding nearby weredas, zones and region population (Projected population of Mekelle in 2008). These both governmental and private health institutions provides health care services on prevention and control of communicable disease, and numerous activities on non communicable diseases through providing a diagnostic, preventive, therapeutic and rehabilitative services having a coverage $83 \%$ since 2009 (19,31).

The Ayder Referral Hospital has been provided services to the 8million population in its catchment areas of the Tigray, Afar and Southeastern parts of the Amhara Regional States. As such, the Hospital can be designated as the most advanced medical facility, by all accounts, in the Northern part of the country and that it stands as the second largest hospital in the nation. With the total capacity of about 500 inpatient beds in four major departments and other specialty units, the Ayder Referral Hospital is also used as a teaching hospital for the College of Health Sciences, Mekelle University (19).

Having above 45 specialists in the various areas of medical specializations and fairly adequate numbers of all the other health professionals constituting the health care team, the Hospital is now emerging as a shinning hot spot for advanced medical care and treatment in the Northern parts of
Ethiopia. With the day-by-day rising patient flow that has already exceeded 100,000 per year, the Ayder Referral Hospital has made remarkable achievements in equipping itself with advanced medical equipment, supplies and facilities over the last four years(19).

Situated in the beautiful University campus of Ayder, the Ayder Referral Hospital runs all the specialized/nonspecialized hospital services including medical, surgical, emergency, laboratory, pharmacy, X-ray, and pathology while preparations are underway to launch postmortem/forensic medicine services (19).

The data was collected from June-September/2014.

\subsection{Study Design}

Institutional based cross sectional study design was undertaken to assess the prevalence and associated factors of CL.

\subsection{Population}

\subsubsection{Source Population}

All patients who come to dermatology OPD.

\subsubsection{Study Population}

All pts who are selected through systemic random sampling methods from those patients come from Mekelle city.

\subsection{Eligibility Criteria}

\subsubsection{Inclusion Criteria}

All patients who comes to the dermatology OPD from Mekelle city during the study period regardless of age and sex.

\subsubsection{Exclusion Criteria}

All pts. who are;

- Patients who are admitted to dermatology ward before data collection and newly admitted patients after participating in the study as a study subject.

- On follow up after pop up as initial study subjects

- Out of the study area i.e. Mekelle city were excluding from this study.

\subsection{Sample Size Calculation and Sampling Procedure}

\subsubsection{Sample Size Determination}

Sample sizes were determined based on previous prevalence of the case $(4.82 \%$ from Siliti, Ethiopia) with level of significance $\alpha 0.05$ desire precision and considering $10 \%$ of non-respondent rate. $\mathrm{n}=\mathrm{Z}(\alpha / 2)^{2} \mathrm{P}(1-\mathrm{P}) / \mathrm{d}^{2}$, Where:

$\mathrm{n}=$ Sample size

$\mathrm{d}=$ precision (marginal error)

$\mathrm{Z}(\alpha / 2)=$ critical value (confident limit)

$\mathrm{P}=$ previous prevalence of $\mathrm{CL}$

Using a reduced margin of error:

Using a reduced margin of error, the calculated sample size having $\mathrm{d}=0.02$, gives;

Thus $\mathrm{n}=(1.96)^{2} \mathrm{X}(0.048)(1-0.048) /(0.02)^{2}$

Total sample size $=437.5 \approx 438$ 
In addition, considering $10 \%$ of non-respondent rate $=$ $10 \% 438=43.8 \approx 44$

Thus, the final sample size were $=437+44=481$

\subsubsection{Sampling Procedure}

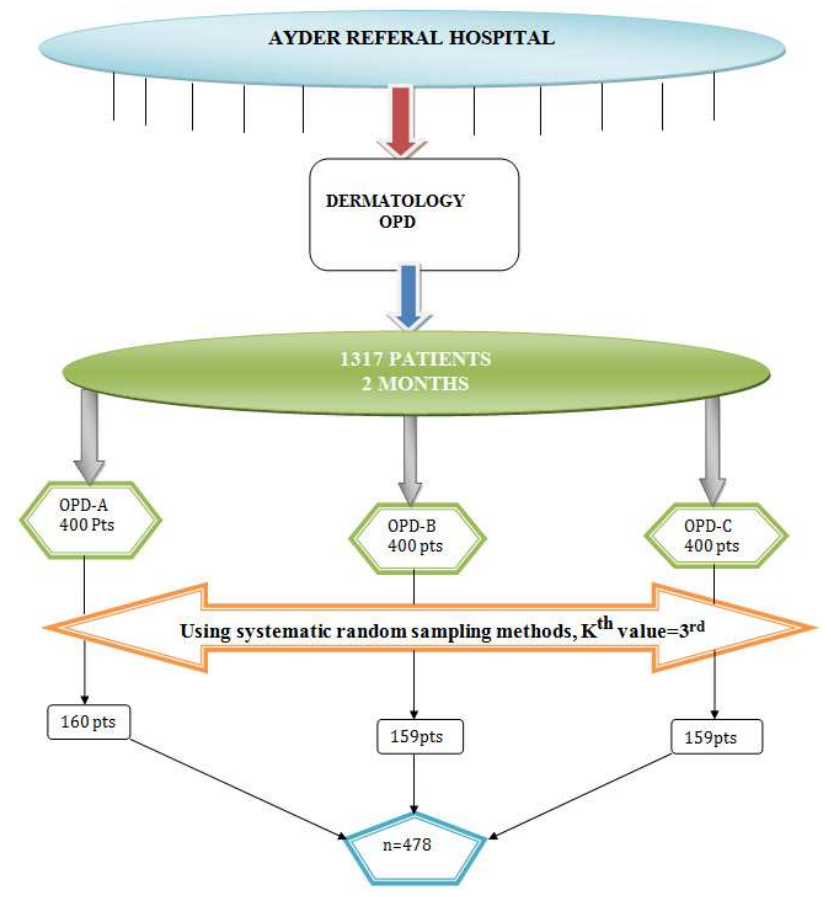

Figure 2. Show techniques of data sampling procedures

Ayder referral hospital is the only regional referral hospital where better number of dermato-venerology field specialist are providing services for the regional population and to the nearby other regions patients. Thus, most patients with CL come to the regional referral hospital where the treatment and field specialist are available there. Therefore, considering this situation, the topic under study was conducted in Ayder referral hospital.

Average numbers of pts flow from Mekelle city in the previous two months were 1317 pts from all dermatology OPDs. I.e. around 400 pts from each dermatology OPD (we have 3 OPDs).

Further, within a data collection period i.e two months (44 working days) I have got around 1317 pts who come to dermatology OPD. Moreover, in order to have the calculated sample size $(n=481)$ within two months from 1200 source population, the study population were selected throygh systematic probable sampling methods with a value $\mathrm{k}=3$. Thus, this calculation provides 162 study populations from each OPD and final sum up source population that I have conducted the study were 478 .

Thus, Systematic sampling technique was implemented to conduct this research.

Data Collection Procedures \& Technique

\subsection{Data Collection Tools and Techniques}

Data collection was undertaken from June-august 2006E.c. through clinical observation using a checklist, standardized questionnaire and laboratory investigation.

During data collection 13 data collectors (Msc Tropical dermatology students) as a shifted pattern , 3 clinical nurse professional, one laboratory expert and one PI were employed to collect the data from those patients who come from Mekelle city only to Ayder referral hospital dermatology OPD as if selected through systematic sampling methods.

\subsection{Study Variables}

\subsubsection{Dependant Variable}

Cutaneouse leishmaniasis

\subsubsection{Independent Variable}

- Socio-demographic variables (sex, age, education status, religion)

- Socio-economic variables (economic status, occupation, Housing condition, travel history, use of bed net)

- Clinical variables (Co-morbidities, duration of the lesion, onset of the lesion after travel to somewhere else, clinical types)

- Ecological variables (Presence of reservoir $\{$ Hyrax $\}$, forest condition, presence and absence of gorge and its distance from the house)

\subsection{Operational Definitions}

- Cutaneous leishmaniasis; Leishmaniasis which only affects skin

- Mucocutaneous leishmaniasis; Leishmaniasis which affects skin and mucosa.

- Diffused CL; Leishmaniasis which is not limited to specific body sites like face, hand, mucosa and the like parts of the body.

- Residence-One who live in Mekelle city at least for the last six (6) months.

\subsection{Data Quality Assurance}

In order to keep the data quality, the questionnaires first prepared in English and then translated to Tigrigna again in order to check the consistency of the Tigrigna questionnaire it was re-translated to English by other person who knows the local language very well.

After providing orientation to the data collectors, pre-test were conducted on $23(4.8 \%$ of the sample size) patients who come to dermatology OPD, but it was not included in the final result, then based the result of the pre test, confusing, leading and questions that should be included were reviewed, reorganized and rewrite and necessary corrections were made with selected data collectors.

During a pre-test data collection, all data collectors (13 tropical dermatology students and 3 clinical nurses) were informed/ aware about the questionnaires' purpose, how to collect and confusing things on questionnaires were well understood.

Besides this, the principal investigator was carefully entered and thoroughly cleaned the data before the 
commencements of the analysis.

Ultimately, the PI was review all filled questionnaires and lab findings accordingly.

\subsection{Data Analysis Procedure}

For all categorical variables frequencies and percentages were calculated. In addition, data entry and analysis was conducted by using SPSS v-20. Then the entered data was edited, cleaned and analyzed. Binary logistic regression analysis was made to obtain odds ratio and the confidence interval of statistical associated variables. All variables with $\mathrm{P}<0.05$ in the bivariate analysis and one which were fit in Hosmer and Lemeshow Test goodness fit model were included in the final model/multivariate analysis, accepting $\mathrm{P}$ $\leq 0.05$ as the inclusion level for the independent variables. Finally, the magnitude of association between different variables in relation to the outcome variable was measured by odds ratio with $95 \%$ confidence interval.

\subsection{Ethical Considerations}

Letter of ethical clearance was obtained from Research Ethical Committee of Mekelle University, College of health sciences. Then official letter wrote to the service delivery points/area, i.e. dermatology unit. Moreover, consent was also obtained from the entire study sample after providing brief information about the objectives and the aim of the research. In addition, all study samples were informed about the participation to this study relies on their will no one can enforced them to participate to the study.

Finally, confidentialities of the information gathered were assured to the interviewee via avoiding the name and address of the interviewee in the questionnaire.

\subsection{Dissemination of the Report}

The result of the study was disseminated to Mekelle University department of Dermatology and Public health.

\section{Result}

During the study period, a total of 1317 clients were visited the dermatolovenereology clinic. Among these, the study was conducted on 486 clients through systematic sampling method with $\mathrm{K}^{\text {th }}$ value of $3^{\text {rd }}$. Meanwhile, 28(5.6\%) had sign and symptoms of cutaneous leishmaniasis on one or more sites of their bodies.

\subsection{Socio-Demographic Characteristics}

Most cases 308(63.4\%) were aged between 16 and 45 years followed by $1-15$ years $154(31.7 \%)$. The mean (SD) age of the clients was $20.89 \pm 12.5 \mathrm{SD}$ years with the age range from 1-72years. The mean age of females and males was $20.8 \pm 12$ and $20.98 \pm 12.92$, respectively. The majority of the respondents were males $258(53.1 \%)$. Most respondents $81.9 \%(n=398)$ were educated while18.1\%( $n=88)$ were illiterate that unable to read and write. Besides, most of the respondents $68.3 \%(n=332)$ were never married yet and the rest $31.7 \%(n=154)$.

Most of the study populations, 94\% $(n=457)$ were Orthodox tewahido followed by Muslim $4.7 \%(n=23)$ and others (protestants and catholic) constitute 6(1.2\%) were participated in the study. Moreover, the majority of the clients 322(66.3\%) were come from four (4) kebeles of Mekelle city, i.e.03, 011, 017 and 018 while the rest $164(33.7 \%)$ of the patients were from the rest kebeles of the city.

In addition, $36.62 \%(\mathrm{n}=178)$ of the respondents were civil servants, $27.57 \% \quad(n=134) \quad$ merchants, $29.1 \% \quad(n=141)$ unemployed including house wife, students and children and only $6.8 \%(n=33)$ were farmers who had a farm land near to the home $12(33.33 \%)$, near to the gorge $9(25 \%)$ and near to both home and gorge $15(41.67 \%)$.

Table 1. Socio-Demographic Characteristics of respondents in Ayder referral hospital, Tigray, Northern Ethiopia, 2014 (n=478)

\begin{tabular}{lllll}
\hline Variables \& Respondents & Frequency $(\mathbf{F})$ & & Percent $\mathbf{( \% )}$ & Mean age=20.89 \\
\hline \multirow{3}{*}{ Respondents' age } & $1-15$ & 154 & 31.7 & $\pm 12.5 \mathrm{SD}$ \\
& $16-45$ & 308 & 63.4 & Median=21.00 \\
Sex of the respondents & 46 and above & 24 & 53.1 & 46.9 \\
& Male & 258 & 18.1 & 33.7 \\
Educational status & Female & 228 & 25.9 & 22.2 \\
& Illiterate & 88 & 68.3 & 31.1 \\
Marital status & Grade1-8 & 164 & 0.6 & \\
& Grade9-12 & 126 & 94.0 & 0.4 \\
Religion & Diploma and above & 108 & 0.8 \\
& Never married & 332 & 4.7 \\
Married & 151 & 6.8 \\
Income source & Divorced & 3 & 27.57 \\
& Orthodox & 457 & 36.52 \\
\hline
\end{tabular}




\subsection{Clinical Characteristics of the Respondents}

Most of the respondents $446(n=91.8 \%)$ had no habit to spent their time near or at the gorge at the morning and/or nights but only $40(8.2 \%)$ were had a habit to spent in such areas for feeding a cattle $(n=3)$, guarding the farm $(n=6)$ and both feeding and guarding the farm $(n=6)$.

Most of the study population $97.9 \% \quad(n=476)$ had no history of travel to somewhere but only $2.1 \%(n=10)$ had history of travel to somewhere with average spent period of less than six months $70 \%(\mathrm{n}=7)$ and the rest $30 \%(\mathrm{n}=3)$ more than six months with presence of the lesion $90(n=9)$ after travel and 10(n=1) before travel somewhere out of Mekelle.

Moreover, $61.9 \%(n=301)$ of the respondents' house floor was carpeted with cement and $38.1 \%(n=185)$ dusted with cow dung/mud having cracked wall/hall $169(\mathrm{n}=34.8 \%)$ and no cracked wall $317(65.2 \%)$ but $191(39.3 \%)$ respondents had animal and animal dung in and/or around their house.

Out of 478 of the study population, $246(50.6 \%)$ had acacia and eucalyptus, $106(21.8 \%)$ eucalyptus, $8(1.6 \%)$ acacia and the rest $126(25.9 \%)$ had other types of tree around their home. On other ways, $8.2 \%(n=40)$ of the respondents had a habits sleeping and sitting in the yard and the rest $91.8 \%(n=446)$ had no such a habits too but $386(79.4 \%)$ of the respondents had no a habits of use of a bed nets whereas the rest $20.6 \%(n=100)$ had a habits to use a bed nets during sleeping at anywhere. In addition, $17.9 \%(n=87)$ of the respondents had habits of working at the morning whereas the rest $82.1 \%(n=399)$ had no such activities.

Among the study population, $28.25 \%(n=137)$ were never tested whereas the rest $71.8 \%(n=349)$ were tested for HIV/AIDS with $2.1 \%(\mathrm{n}=10)$ study population reactive for HIV/AIDS and from these $5.7 \%$ of the study population had co-infection with cutaneouse leishmaniasis but the rest $69.8 \%(n=339)$ were non-reactive.

A total of $14 \%(n=68)$ of the respondents live in the area where termite hills are there and the rest $86 \%(n=418)$ live in the area free of termite hill. On the other ways, $57(11.7 \%)$ respondents lived in the area rich in hyrax with $11.3 \%(\mathrm{n}=55)$ increase its population through time and $7.8 \% \quad(n=38)$ respondents saw the hyrax, which moves to their residents home, but $429(88.3 \%)$ respondents lived in area free of hyrax.

\section{Presence Of Clinicaly Cutaneous Leishmaniasis}

Among the study population, 28(5.6\%) of the clients had clinically cutaneous leishmaniasis of which $62.9 \%(n=22)$ presented on single body sites and the rest $37.1 \%(n=13)$ found on multiple body sites on face other than mucosa $16(45.7 \%)$, mucosa (lip, nasal tip, eye) 16(45.7\%), and $8.6 \%(\mathrm{n}=3)$ of the lesion found on the extremities from which $31.4 \%$ were positive for slit skin smear the rest $68.6 \%$ were negative for SSS even though clinically diagnosed as localized cut leishmaniasis $51.4 \% \quad(\mathrm{n}=18)$, muco-cutaneous leishmaniasis $25.7 \% \quad(n=9)$ and diffused cutaneous leishmaniasis $22.9 \%(\mathrm{n}=8)$.

Besides, 12 (2.5\%) of the respondents had previous scare due to "guzua" whereas the majority of the respondents 474(97.5\%) were free.

\section{Percentages(\%)}

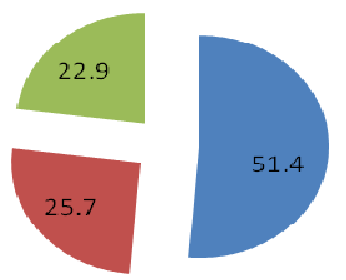

- Localized cutaneouse leishamniasis

Muco-cutaneouse leishmanisis

Diffused cutaneouse leishamniasis

Figure 3. Pie chart show types of cut leishmaniasis on the respondents Ayder referral hospital, Tigray, 2014(n=478)

Table 2. Clinical factors to the presence of cutaneouse leishmaniasis in Ayder Referral Hospitals, Tigray, Northern Ethiopia, 2014(n=478)

\begin{tabular}{|c|c|c|}
\hline Variables and respondents' response & Frequency $(\mathbf{F})$ & Percents $(\%)$ \\
\hline \multicolumn{3}{|l|}{ Common trees around home } \\
\hline Acacia & 8 & 1.6 \\
\hline Eucalyptus & 106 & 21.8 \\
\hline Acacia \& eucalyptus & 246 & 50.6 \\
\hline Others & 126 & 25.9 \\
\hline \multicolumn{3}{|l|}{ Presence of termite hill } \\
\hline Yes & 68 & 14.0 \\
\hline \multicolumn{3}{|l|}{ Presence of hyrax } \\
\hline Yes & 57 & 11.7 \\
\hline No & 429 & 88.3 \\
\hline \multicolumn{3}{|l|}{ Number of the lesion over the respondents } \\
\hline Single & 22 & 62.86 \\
\hline Multiple & 13 & 37.14 \\
\hline \multicolumn{3}{|l|}{ Clinical forms of cutaneous leishmaniasis } \\
\hline DCL & 8 & 22.9 \\
\hline \multicolumn{3}{|l|}{ Slit skin smear result } \\
\hline Positive & 11 & $31.4 \%$ \\
\hline Negative & 24 & $68.6 \%$ \\
\hline
\end{tabular}




\subsection{Factors Associated With Presence of Cutaneous Leishmaniasis}

\section{Bivariate Analysis}

Determination of all independent variables to the factors for the presence of cutaneouse leishmaniasis was made by logistic regression model with the assumption that it helps to predict the extent by which this outcome variable could be explained by independent variables. All variables which had $\mathrm{p}<0.05$ on the bivariate analysis after goodness fit model was entered in to multivariate analysis.

Thus, through bivariate logistic regression ten independent variables, i.e., age, sex, income source, location of the home, time of spent near or/and to the gorge, house condition of the floor, wall, working habit at the morning/evening, presence of termite and hyrax were shown a significant association to the presence of cutaneouse leishmaniasis.

The odds of having cutaneouse leishmaniasis on males with respect to females study population were found to be 2.7 with $\{\operatorname{COR}(95 \% \mathrm{CI}) ; 2.7(0.167,0.793)\}$, income source farming $\{\mathrm{COR}(95 \% \mathrm{CI}) ; 13.8(1.044,0.13)\}$, with respect to daily laborers, house wives and others, location of the home near to both to the gorge and farm land $\{\mathrm{COR}(95 \% \mathrm{CI}) ; 49.3$ $(0.029,0.515)\}$ with respect to the residence area far away from gorge and farm land and presence of hyrax with $\{\mathrm{COR}(95 \% \mathrm{CI}) ; 2.7(0.005,0.037)\}$ with respect to hyrax free area.

Then, after testing in to the model goodness fitness test these data were re-entered in to the multivariate analysis.

\section{Multivariate Analysis}

The odds of having cut leishmaniasis among male participants was 2.11 compared to female participants with $\{$ AOR $(95 \% \mathrm{CI}) ; 2.11(0.015,0.793)\}$ whereas the odds of having cut leishmaniasis among respondents whose home situated around/near farm land and/or gorge compared to those who lived far/free from farm and gorge was 10.10 with \{AOR $(95 \% \mathrm{CI}) ; 10.11(0.00,0.574)\}$, on other way, the odds of having cutaneouse leishmaniasis among farmers were 2.1 $(0.00,0.248)$, with $95 \% \mathrm{CI}$ and finally the odds of having cut leishmaniasis among respondents who lived around hyrax population compared to respondents who lived hyrax free area was 1.101 with $\{\mathrm{AOR}(95 \% \mathrm{CI}) ; 1.1(0.00,0.10)\}$. But presence of termite hill, working time at the morning and/or nights, age and housing conditions (floor and wall) characteristics/variables had not significant association with presence of cut leishmaniasis through Multivariate analysis.

Table 3. Factors associated with the presence of cut leishmaniasis in Ayder referral hospiral, Tigray, Northern Ethiopia, 2014 (n=478).

\begin{tabular}{|c|c|c|c|c|c|}
\hline \multirow{2}{*}{ Variables } & & \multicolumn{2}{|c|}{ Cutaneous leishmaniasis } & \multicolumn{2}{|l|}{ OR $(95 \% \mathrm{CI})$} \\
\hline & & Yes & No & COR & AOR \\
\hline \multirow{3}{*}{ Sex } & Male & 26 & 232 & $2.72(0.167,0.793)$ & $2.108(0.15,0.793)$ \\
\hline & Female & 9 & 219 & 1 & - \\
\hline & Near to the farm & 6 & 9 & $32.84(0.030,0.276)$ & $6.14(0.00,0.96)$ \\
\hline \multirow{3}{*}{$\begin{array}{l}\text { Location } \\
\text { of the home }\end{array}$} & Near to the gorge & 7 & 9 & $25.54(0.000,0.17)$ & $0.034(0.0,0.23 .07)$ \\
\hline & Near to both $1 \& 2$ & 9 & 6 & $49.27(0.029,0.515)$ & $10.10(0.00,0.574)$ \\
\hline & No & 13 & 427 & 1 & - \\
\hline \multirow{3}{*}{$\begin{array}{l}\text { Income } \\
\text { source }\end{array}$} & Merchants & 9 & 125 & $1.35(7.60,20.82)$ & $0.13(0.00,0.505)$ \\
\hline & Civil servants & 9 & 169 & 1 & - \\
\hline & Unemployed & 11 & 130 & $1.59(2.41,10.41)$ & $0.16(0.001,0.40)$ \\
\hline \multirow{2}{*}{ Termite } & Yes & 24 & 44 & $20.18(0.019,0.096)$ & $1.36(0.204,9.037)$ \\
\hline & No & 11 & 407 & 1 & - \\
\hline \multirow{2}{*}{ Hyrax } & Yes & 23 & 34 & $23.5(0.005,0.037)$ & $1.101(0.00,0.60)$ \\
\hline & No & 12 & 417 & 1 & - \\
\hline \multirow[t]{2}{*}{ Age } & 16-45years & 24 & 282 & $0.322(1.99,8.27)$ & $0.344(0.22,5.48)$ \\
\hline & 46 and above & 5 & 19 & 1 & $\ldots$ \\
\hline
\end{tabular}

\section{Discussion}

This study was tried to assess the prevalence and associated factors of cutaneous leishmaniasis in institutional level. It was aimed to determine the prevalence and identify associated factors of cutaneous leishmaniasis with specific socio-demographic data of the study population such as sex, source of income, place of residence, educational status, occupation and the likes. Thus, the prevalence of cutaneous leishmaniasis in the study area was $5.6 \%$ with associated factors of sex, location of the farm, income source, and hyrax.

The prevalence of cutaneous leishmaniasis in the study area was $5.6 \%$, which was relatively higher finding compared to other similar studies conducted in Tigray regions (4.8\%), Siliti (4.82\%), Ochollo (3.6-4.0\%), and Sebetta $(0.9 \%)$. This is due to the other similar studies were conducted as a community based (level), most of the population $79.4 \%$ of the study population had no a habits to use a bed nets during sleeping at anywhere, the topography of the area is found in higher altitude (more than $2000 \mathrm{~m}$ above sea level), nature of the soil (clay and sandy) which provides the best habitats for the vectors sand fly, presence of hyrax $(13.17 \%)$, the difference in diagnostic methods in that this study was determined by using clinically diagnosing methods and/or skin slit smear (which where only $31.4 \%$ were positive) but the other study areas were used including multiple sophisticated diagnosing methods like PCR and the 
presence of vector habitats to human dwellings, human activities (such as firewood collection, herding domestic animals, land use) and environmental factors, including vegetation type like could be a basic reason that make the study as such out numbered.

In this study gender had significant association to the prevalence of cutaneous leishmaniasis. The odds of having cutaneous leishmaniasis on male gender were \{AOR $(95 \% \mathrm{CI}) ; 2.11(0.015,0.793)\}$. This implies the possibility of having cutaneous leishmaniasis was relatively higher more twice fold as males than females. This was similar to the study conducted in Colombia, Siliti(47.8\% male; $52.2 \%$ female), India (M:F, 75.93\%: 24.07\%) and Jordan (M:F, $72.40 \%: 27.60 \%)(4,18,22)$. This might be due to males appear to have a higher prevalence probably because of environmental exposure to the habitat of the sand fly through occupation and leisure activity, as there is no significant difference in gender attendance to health services and our cultural activities that mostly males are faced with outdoor activities including farming, keeping cattle, stayed around the gorge and/or farm for long period and the presence of endemic site(s) like Humera and Metema that mostly males could travel there for work $(19,25)$. But to the contrary a study conducted in America stats no significant difference was found between males and females (M:F, 52\%:48\%) suggesting that they are equally exposed to infection. This clearly tells us the variation in source of income (Americansindustry vs Ethio-\& the like agriculture) and the nature of the activities that exposes males for sandfly than females. In this study these my findings could be signified by $31.42 \%$ ) of the cases were found on farmers than civil servants or merchants.

Being a farmer had a significant association to the presence of cutaneouse leishmaniasis with the odds of $\{$ AOR $(95 \% \mathrm{CI}) ; 2.102(0.000,0.248)\}$. This meant that being a farmer had a relative higher exposure to acquire cutaneous leishmaniasis as nearly twice $(2 \mathrm{x})$ fold to contract the disease than daily workers, house wives and students. This finding was incomparably higher relative to a study conducted in America. This is probably due to the difference in source of income, i.e. in America is mostly the source of income is industry to the contrary of our country but almost similar finding to that of a study conducted in India and Siliti due to a similar in source of income and cultural activities.

The presence of hyrax around the respondents' home had a significant association to the prevalence of cutaneous leishmaniasis. The odd of having cutaneous leishmaniasis in the area with hyrax was $\{$ AOR $(95 \% \mathrm{CI}) ; 1.101(0.000,0.10)\}$. This result showed that the population they settle in areas where rich in hyrax colony had a relative higher exposure to have cutaneous leishmaniasis than hyrax free areas. This was similar to the study conducted in Kenya, Uganda and Addis Ababa $(8,32,33)$ and relatively higher to a study conducted in Siliti this is due to the presence of forests in the city like acacia $(1.6 \%)$, eucalyptus $(21.8 \%)$, and both $(50.6 \%)$, clay soil and sandy area with associated with higher altitude provides a good habitat for the hyrax which is a good harbor for sand fly but to the contrary to the study conducted in
Meta Abo Ethiopia these characteristics had no any significant association (30).

Ultimately, location of the home both to the farm and gorge had a significant association to the presence of cutaneouse leishmaniasis with the odds of A AOR $(95 \% \mathrm{CI})$; $10.11(0.000,0.574)\}$. This was relatively higher with the previous reports from America (Argentina) with A AOD (95\% $\mathrm{CI}): 2.6(1.2,5.5)\}$ to the farm land and AAOD $(95 \%$ CI): $15.1(6.1,7.3)\}$ to the gorge and Siliti A AOD (95\% CI):1.762 $(2.6,5.179)\}$ area $(4,15)$. This could be due to the potential to have an impact on the sandfly population density and provide suitable conditions for sandfly breeding sites (low temperature, moderate humidity, and presence of living organic matter).However, in this study both the presence of gorge and farmland around the respondents' home had a relative weak association with respect to both studies. This might be due to difference in the study population (Siliticommunity based study) and study design (ArgentinaCohort study).

\subsection{Strength of the Study}

In this study the main strengths were; using primary data.

\subsection{Limitation of Study}

- The study strongly affected by selection bias because the study preferably good if it was conducted as a community level/base.

- Result may not generalizable to other areas outside the study area.

- The study does not show cause and effect relationship.

\section{Conclusion and Recommendation}

\subsection{Conclusion}

The finding in this study in relation to the presence of cutaneous leishmaniasis revealed that the prevalence of cutaneous leishmaniasis in the study area was $5.6 \%$ which were too high relative to the study conducted yet. Most males and farmers are groups to have relatively higher exposure for cutaneous leishmaniasis with respect to the location of farmland and gorge with the presence of hyrax.

\subsection{Recommendations}

Based on the findings from this study the following recommendations are made:

\section{To the Health Proffessionals}

Provide regular health education about the factors, mode of transmission and preventive modalities of coetaneous leishmaniasis.

\section{To Mekelle City Health Office}

One of the factors for the presence of cutaneouse leishmaniasis was the presence of gorge and hyrax. Thus, the city health office should:

- Preferably remove or kill all hyrax from the community.

- Facilitates the health professionals to provide health 
education about the factors, mode of transmission and preventive modalities of cutaneouse leishmaniasis.

- Make all gorge to be labeled.

\section{References}

[1] Bailey MS, Lockwood DNJ. Cutaneous leishmaniasis. Clinics Dermatol 2007; 25:203-11.

[2] Blum JA, Hots CF. Treatment of cutaneous leishmaniasis in travelers. J Travel Med 2009; 16:123-31.

[3] Postigo JA. Leishmaniasis in the World Health Organization Eastern Mediterranean Region, Int J Antimicrobial Agents, 2010; 36: S62-5.

[4] Negera, Gadisa, Yamuah, et el. outbreak of cutaneous leishmaniasis in Silti woreda,Ethiopia: risk factor assessment and causative agent, transactions of the royal society of tropical medicine and hygiene, 2008, 102, 883-890

[5] Ashford, R.W.The leishmaniases as emerging and reemerging zoonoses.Internat.J.Parasitol. 2000. 30: 1269-1281

[6] Cooke, J.G. Public Health in Africa: A Report of the CSIS Global Health, 2009,PolicyCentre.Retrieved from; http://csis.org/files/media/csis/pubs/090420_cooke_pubhealth africa_web.pdf

[7] Control of the leishmaniasis: Report of A meeting of the WHO Expert Committee on the Control of leishmaniases, World Health organization, Geneva, Switzerland, 2010.

[8] Abyot D, Solomon S, Andargachew K, et el, Modules on leishmaniasis, Carter center Ethiopia, 2005,11-13.

[9] Wilkins, H.A. Studies on leishmaniasis in Ethiopia: incidence rates of cutaneous leishmaniasis at Meta Abo. Ann.Trop.Med.Parasitol. 1972, 66:457-463

[10] Gebre-Michael, T., Balkew, M., et el, The isolation of Leishmania aethiopica and Leishmania tropica from Phlebotoms (paraphlebotomus) species (Diptera: Psychodidae) in Awash Valley, N. Ethiopia. Transc.R.Soc.Tro.Med.Hyg. 2004. 98:64-70.

[11] Hailu, A., Abebe T., Hunegnaw et al Isolation of Leishmania tropica from an Ethiopian cutaneous leishmaniasis patient. Trans. R.Soc. Trop. Med. Hyg. (2006). 100: 53-58.

[12] Memórias do Instituto Oswaldo Cruz - Epidemiology of leishmaniasis in Ecuador: current status of knowledge, Mem. Inst. Oswaldo Cruz vol.99 no.7 Rio de Janeiro Nov. 2004

[13] PedrosaFde A, et al: Socio-demographic and environmental risk factors for American cutaneous leishmaniasis (ACL) in the State of Alagoas, Brazil. Am J Trop Med Hyg2009, 81(2): 195-201.

[14] Lane RP. Phlebotomine sandflies. In: Manson P, Cook GC, Zumla A, eds. Manson's Tropical diseases. 21st ed. London: Saunders, 2003:1733-41

[15] .Rabello A Leishmania/HIV co-infection in Brazil: role of the national network for surveillance. In: Proc. Third World Congress on Leishmaniasis, 10-15 April, Palermo-Terrasini, Brazil, 2005.
[16] Desjeux, P. The increase in risk factors for leishmaniasis world wide. Trans. R. Soc. trop. Med.and Hyg. 2001, 54: 239243.

[17] Padovese V,Terranova M, Toma L, et al, A Epidemiological and geographical aspects of leishmaniasis in Tigray, northern Ethiopia: a retrospective analysis of medical records, 20052008.Royal Societyof Tropical Medicine and Hygiene. Published by Elsevier Ltd, Copyright (c) 2011

[18] Hattingh, S. P., Dreyer, M., \& Roos, S, Aspects of Community Health. South Africa: Oxford University Press, 2006, 246.

[19] WWW.Wikipidia.tigiray region

[20] WHO Control of the leishmaniases. Report of a meeting of the WHO Expert Committee on the Control of Leishmaniases, 22-26 March 2010, Geneva 5-88.

[21] Leishmaniasis. (n.d.). World Health Organization. Retrieved July 9, 2012, from http://www.who.int/leishmaniasis/en/

[22] Raúl H. Pardo, Alexander Carvajal and et el ,Effect of knowledge and economic status on sandfly control activities by householders at risk of cutaneous leishmaniasis in the subandean region of Huila department, Colombia, vol.26 suppl.1 Bogotá Oct. 2006

[23] Bora. The national medical journals of India vol. 12, no.2, 199948/ Eshete G, Sonder K, ter Heegde F. Report on the feasibility study of a national program for domestic biogas in Ethiopia. 2006 http://www.snvworld.org/en/Documents/NDBPfeasibility study Ethiopia 2006.pdf [accessed 11 February 2011].

[24] Bashaye S, Nombela N, Argaw et al. Risk factors for VL in a new epidemic site in Amhara region, Ethiopia. Am J Trop Med Hyg, 2009, 81: 34-39.

[25] Khalid FA. et al., Treatment of Cutaneous leishmaniasis with some Local Sudanese Plants (Neem, Garad\& Garlic), Türkiye Parazitol oji Dergisi 28 (3): 129-132 2004.

[26] Al-Jawabreh, A., et al, Epidemiology of cutaneous leishmaniasis in endemic area of Jericho, Palestine. East. Mediterran. J.Heal. (2003), 9: 805-815.

[27] Alvar J, Aparicio P, Aseffa A, Den Boer M, Cañavate C et al (2008). The Relationship between Leishmaniasis and AIDS: the Second 10 Years. Clin Microb Rev 21(2):334-359.

[28] WHO report on global surveillance of epidemic--prone infectious diseases, 2000; Retrieved from; http://www.who.int/emc

[29] Zaida E. Yadon, Laura C. Rodrigues, Clive R. Davies, and et al: Indoor and peri-domestic transmission of American cutaneous Leishmaniasis in Northwestern Argentina: a retrospective case-control study: Am. J. Trop. Med. Hyg., 68(5), 2003, pp. 519-526

[30] Dawit G, Girma Z, Simenew K. A Review on Biology, Epidemiology and Public Health Significance of Leishmaniasis Bacteriol Parasitol, 2013.

[31] Tigray regional state bureau of plan and finance September, 2003 E.c, 28-30. 